\title{
Flash pyrolysis-gas chromatography of the kerogen and asphaltene fractions isolated from a sequence of oil shales $^{\text {ش }}$
}

\author{
J.C. del Río*, J. García-Mollá, F.J. González-Vila and F. Martín \\ Instituto de Recursos Naturales y Agrobiologia de Sevilla, CSIC, P.O. Box 1052, 41080-Seville (Spain)
}

(First received December 10th, 1992; revised manuscript received April 24th, 1993)

\begin{abstract}
Molecular characterization of the kerogen and asphaltene fractions isolated from three oil shales taken at increasing depth from the same deposit was carried out by means of flash pyrolysis-gas chromatography. Pyrolysis products were detected by simultaneous flame ionization detection and sulphur-selective flame photometric detection using a stream splitter at the end of the capillary column. $n$-Alkanes, $n$-alk-1-enes and sulphur compounds represent the major pyrolysis products of both kerogen and asphaltene fractions. Organosulphur compounds were only detected in the kerogen fractions and were completely absent from the asphaltene fractions. A sequential removal of thiophenes was observed in the set of kerogens. It is suggested that breakdown of sulphur-sulphur and carbon-sulphur bonds may occur preferentially in the kerogen macromolecule rather than the breakdown of carbon-carbon bonds to give rise to the asphaltenes.
\end{abstract}

\section{INTRODUCTION}

As is well known, knowledge of the chemical nature of asphaltenes and kerogens may help in a better understanding of the source of precursor materials, the environmental conditions of deposition and the type of diagenetic, catagenetic and maturation processes.

Flash pyrolysis-gas chromatography (Py-GC) is one of the most important analytical tools for the structural characterization of insoluble organic materials present in such samples. Hydrocarbons produced by pyrolysis of kerogens and their precursors have been investigated for many years $[1-3]$.

\footnotetext{
* Corresponding author.

"Presented at the 21st Scientific Meeting of the Spanish Group of Chromatography and Related Techniques, Granada, October 21-23, 1992.
}

Today, an important goal in fossil fuel geochemistry is the determination of the amounts and types of organically bound sulphur in sedimentary organic matter since sulphur moieties play a key role in polycondensation processes leading to macrostructures such as kerogens. Sulphur compounds are well known constituents of fossil macromolecules and occur in a variety of molecular structures, including aliphatic thiols, mono- and disulphides and aromatic structures such as alkylated thiophenes, benzothiophenes and dibenzothiophenes [4,5]. In this work we study the pyrolysis behaviour of kerogens, and their related asphaltenes, isolated from a set of Spanish oil shales, in order to get insight into the structure of these materials. We focused our interest on the nature of the sulphur compounds produced in the pyrolysis using simultaneous flame ionization detection (FID) and sulphur-selective flame photometric detection (FPD). 


\section{EXPERIMENTAL}

The oil shale samples were taken from the Puertollano (Ciudad Real, Spain) deposit. The geological setting of this oil shale, from the Stephanian B, has already been described [6]. Three samples were collected at the Emma Mine, at increasing depth (Emma A, B and C).

Raw oil shale samples were milled and extracted exhaustively with methylene chloridemethanol (1:1). Minerals were removed by successive treatments with $20 \% \mathrm{HCl}\left(12 \mathrm{~h}, 60^{\circ} \mathrm{C}\right)$ and $\mathrm{HCl}-\mathrm{HF}(1: 1)\left(24 \mathrm{~h}, 60^{\circ} \mathrm{C}\right.$, twice). The leached material was extensively washed and extracted with water until it was free of chloride ions. The dried residues (kerogens) were then analysed by flash Py-GC. Alternatively, aliquots of the oil shale extracts were dissolved in a minimum amount of chloroform and the asphaltene fractions precipitated with $n$-pentane. Asphaltenes were separated by centrifugation, and the pentane subsequently removed by decanting. The precipitation process was repeated twice in order to purify the asphaltenes.

The samples $(20-40 \mathrm{mg}$ ) were pyrolysed at a temperature setting of $800^{\circ} \mathrm{C}$ for $20 \mathrm{~s}$, using a Chemical Data System (CDS) Pyroprobe 122 system. The pyrolysates were rapidly removed from heated interface $\left(300^{\circ} \mathrm{C}\right)$ by a stream of helium onto a $30 \mathrm{~m} \times 0.25 \mathrm{~mm}$ I.D. fused-silica capillary column coated with DB-5 (J \& W Scientific) in a Varian 3300 gas chromatograph equipped with an efluent splitter and both flame ionization and flame photometric detectors. The column temperature was held at $-25^{\circ} \mathrm{C}$ for $4 \mathrm{~min}$ and raised to $300^{\circ} \mathrm{C}$ at a rate of $4^{\circ} \mathrm{C} / \mathrm{min}$. Helium was used as carrier gas. Tentative identification of the FPD peaks was made by comparison with chromatograms already published. Differentiation of some isomer clusters was also accomplished by GC-MS analyses.

\section{RESULTS AND DISCUSSION}

The kerogen and asphaltene fractions isolated from the selected oil shale samples were pyrolysed and the thermal degradative products analysed on-line by GC with simultaneous FID and FPD. Fig. 1 shows the FID and FPD

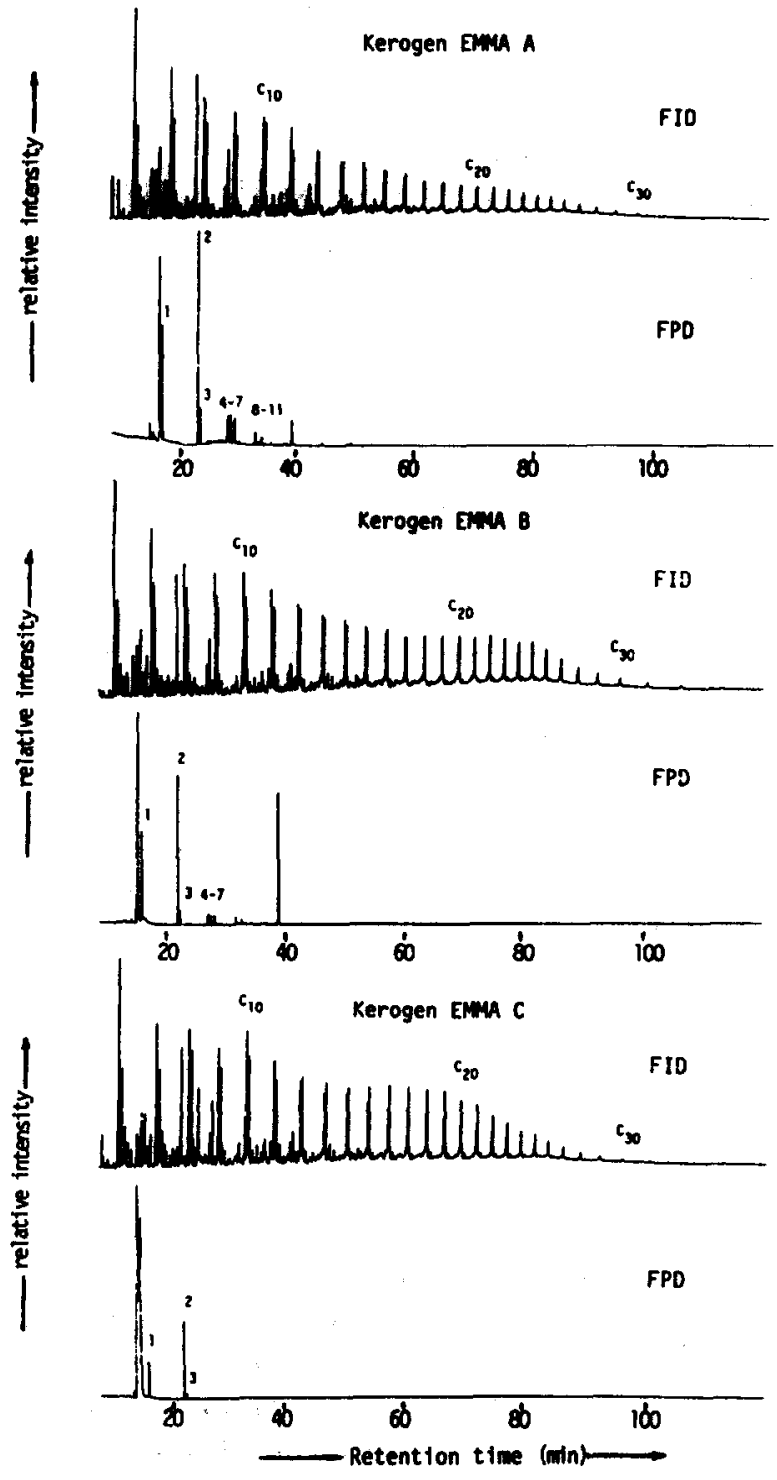

Fig. 1. Flash pyrolysis-gas chromatograms (FID and FPD traces) of the kerogen fractions isolated from the Puertollano oil shales Emma $A, B$ and $C$ (numbers in the FPD trace refer to the organosulphur compounds listed in Table I).

chromatograms of the kerogen pyrolysates. Peak identifications for the sulphur compounds in the FPD trace are reported in Table $I$. The pyrograms were dominated by a series of doublets consisting of $\boldsymbol{n}$-alk-1-enes and $\boldsymbol{n}$-alkanes ranging up to $C_{31}$. This fact confirms the high aliphatic character of the kerogen fraction isolated from the Puertollano oil shale. This character probably arises from a highly aliphatic and resistant 


\section{TABLE I}

\section{SULPHUR COMPOUNDS IDENTIFIED IN THE PYROLYSATES}

\begin{aligned} & \hline 1 Thiophene \\ & 2 2-Methylthiophene \\ & 3 3-Methylthiophene \\ & 4 2-Ethylthiophene \\ & 5 2,5-Dimethylthiophene \\ & 6 2,4-Dimethylthiophene \\ & 7 2,3-Dimethylthiophene \\ & 8 2-Ethyl-5-methylthiophene \\ & 9 2-Ethyl-4-methylthiophene \\ & 10 2,3,5-Trimethylthiophene \\ & 11 2-Methyl-5-vinylthiophene \\ & \hline\end{aligned}

biomacromolecule of present-day and extant organisms that has been selectively preserved during diagenesis [7]. These biomacromolecules can explain the high aliphatic character of the kerogen fractions studied here, and the series of $n$-alkanes and $n$-alk-1-enes produced in the pyrolysis. A slight trend towards the production of higher hydrocarbons was observed in going from sample $A$ to $B$ and $C$. The main aromatic compounds identified in the pyrolysates (benzene, toluene and xylenes) were only found in minor amounts. Prist-1-ene, thought to be derived from the thermal breakdown of macromolecularly bound tocopherols [8], was not found in any of the kerogen pyrolysates.

The FPD pyrograms of the kerogen fractions revealed that thiophene and $\mathrm{C}_{1}-\mathrm{C}_{3}$ alkylated thiophenes were the major sulphur-containing pyrolysis products. Hydrogen sulphide was not produced in the pyrolysis, indicating the absence of sulphide and/or polysulphide moieties in the kerogen fractions isolated from the Puertollano oil shales. It may be possible that these moieties are lost in preference to thiophenic structures.

Each alkylthiophene cluster presents a limited number of isomers. This is an indication that the thiophenic compounds present in the kerogen would be formed from breakdown of pre-existing thiophenic molecules in the kerogen and not from reaction of sulphur (or $\mathrm{H}_{2} \mathrm{~S}$ ) with organic radicals during pyrolysis. The kerogen fractions isolated from the Puertollano oil shale showed a predominance of compounds derived from moi- eties with linear carbon skeletons (2-alkyl- and 2,5-dialkylthiophenes). Nevertheless, the contribution of branched/isoprenoid structures (2,3dialkyl- and 2,3,5-trialkylthiophenes) is considerable, as they correspond to nearly $15 \%$ of the alkylthiophenes.

The organically bound sulphur is probably formed by incorporation of inorganic sulphur species into functionalized precursor lipids during early diagenesis. The sulphur compounds are incorporated into kerogens, which with increasing maturity become more aromatic and are released upon pyrolysis as aromatic thiophenes.

The FID pyrograms of the asphaltenes isolated from the same oil shales are shown in Fig. 2 . They show a predominance of hydrocarbons extending up to $\mathrm{C}_{35}$, further than in the kerogen pyrolysates. However, examination of the FPD pyrograms of the asphaltene fractions (not shown here) showed that no sulphur compounds were present. This would suggest that breakdown of the kerogen may occur preferentially at the sulphur bridges. It may be possible that the preferential breakdown of the thiophene bridges present in the macromolecular structure of the kerogen will release smaller fragments, giving rise to the formation of asphaltene moieties, in

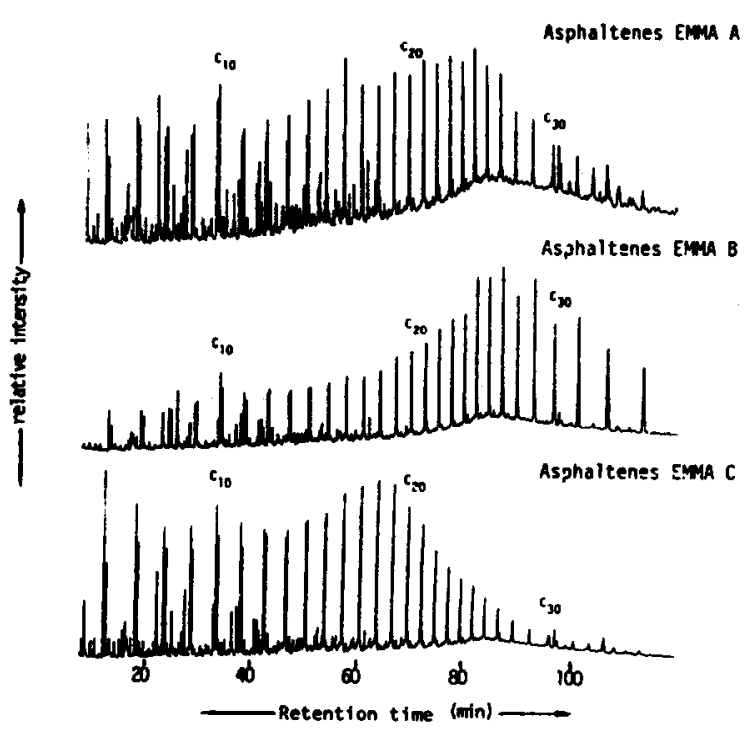

Fig. 2. Flash pyrolysis-gas chromatography (FID trace) of the asphaltene fractions isolated from the Puertollano oil shales Emma A, B and C. 
agreement with the fact that asphaltenes are thought to be essentially small solubilized kerogen fragments [9]. In fact, sulphur-sulphur and sulphur-carbon bonds are weaker than carbon-carbon bonds $[10,11]$. This result would suggest that the S-containing moieties, as thiophenes, are mainly bound to the macromolecular matrix by $\mathrm{C}-\mathrm{S}$ bonds. The presence of these weak bonds lowers the maximum of the activation energy distribution for the thermal degradation. Thus, it is well known that sulphurrich kerogens exhibit a much lower Rock-Eval $T_{\max }$ than sulphur-poor kerogens of the same maturity $[12,13]$. These results would support the idea that the organic sulphur is, at least partially, responsible for the early petroleum generation from S-rich kerogens. Organically bound sulphur has been implicated in the formation of low-APIgravity $^{a}$, asphaltene-rich petroleums at a low level of thermal exposure [11].

\section{CONCLUSIONS}

The kerogen and asphaltene fractions isolated from a set of oil shales from the Puertollano deposit were analysed by flash pyrolysis-gas chromatography with simultaneous FID and FPD. This technique proved to be useful in getting insight into the macromolecular structure of the geopolymers (asphaltene and kerogen) isolated from the selected oil shales. The main compounds produced were series of $n$-alkanes and $n$-alkenes arising probably from the presence of a highly aliphatic and resistant biopolymer in their structure. Sulphur compounds were only detected in the pyrolysis of the kerogens, sug-

\footnotetext{
a API = American Petroleum Institute. ${ }^{\circ}$ API gravity is a measure of the specific gravity of a crude oil.
}

gesting that sulphur-sulphur and sulphur-carbon bonds may be preferentially broken down rather than the carbon-carbon bonds in the kerogen macromolecule to give rise to the asphaltenes. A lack of $\mathrm{H}_{2} \mathrm{~S}$ has been observed in the kerogen pyrolysates, suggesting that (poly)sulphide moieties are removed preferentially during diagenesis and maturation, rather than thiophenic moieties.

\section{REFERENCES}

1 D. van de Meent, S.C. Brown, R.P. Philp and B.R.T. Simoneit, Geochim. Cosmochim. Acta, 44 (1980) 999 1013.

2 S.R. Larter, in K.J. Voorhees (Editor), Analytical Pyrolysis Techniques and Explorations, Butterworths, London, 1984, 212-275.

3 R.P. Philp, A. Bakel, A. Galvez-Sinibaldi and L.H. Lin, Org. Geochem., 15 (1988) 915-926.

4 W.L. Orr and C.M. White (Editors), Geochemistry of Sulfur in Fossil Fuels (ACS Symposium Series, No. 429), Americal Chemical Society, Washington, DC, 1990.

5 J.S. Sinninghe Damsté and J.W. de Leeuw, Fuel Processing Technology, 30 (1992) 109-178.

6 R.H. Wagner, Ann. Fac. Cienc., Porto, 64 (1985) 171231.

7 E.W. Tegelaar, J.W. de Leeuw and C. Saiz-Jimenez, Sci. Total Environm., 81/82 (1989) 1-17.

8 H. Goossens, J.W. de Leeuw, P.A. Schenck and S.C. Brassell, Nature, 312 (1984) 440-442.

9 B.P. Tissot and D.H. Welte, Petroleum Formation and Occurrence, Springer, Heidelberg, 2nd ed., 1984.

10 E. Tannenbaum and Z. Aizenshtat, Org. Geochem., 8 (1985) 181-192.

11 W.L. Orr, Org. Geochem., 10 (1986) 499-516.

12 T.I. Eglinton, J.S. Sinninghe Damsté, M.E.L. Kohnen, J.W. de Leeuw, S.R. Larter and R.L. Patience, in W.L. Orr and C.M. White (Editors), Geochemistry of Sulfur in Fossil Fuels (ACS Symposium Series, No. 429), American Chemical Soceity, Washington, DC, 1989, pp. 529-565.

13 T.I. Eglinton, J.J. Sinninghe Damsté, M.E.L. Khonen and J.W. de Leeuw, Fuel, 69 (1990) 1394-1404. 